\title{
Normes religieuses et loi du silence : le mariage temporaire chez les chiites du Liban
}

Sabrina Mervin

Les normes religieuses dont il sera question ici sont énoncées dans les ouvrages de droit islamique (fiqh) et dans la jurisprudence, à savoir les recueils de fatwas. Elles régissent le mariage appelé en arabe zawâj al-mut' $a$ (de jouissance ou de plaisir), ou bien muwaqqat, ou munqati ' (temporaire). Alors que la première expression est ancienne, et fait l'objet d'un emploi courant, et populaire, les deux dernières sont plus récentes et ont la préférence des clercs chiites qui les utilisent par euphémisme. En persan, le terme retenu couramment est sighe, qui renvoie à la notion de contrat et à la formule orale que prononcent les époux.

Cette forme d'union est une spécificité du droit islamique ja'farite (celui des chiites duodécimains), qui n'est pas admise par les quatre écoles juridiques sunnites ${ }^{1}$. La pratique remonte à la période pré-islamique, et elle était en usage au temps du prophète Muhammad avant d'être abolie par le calife 'Umar. Selon un hadîth connu, celui-ci la dénonça et l'interdit car il la considérait comme une forme de prostitution, ce qui incita les chiites, qui avaient un contentieux avec lui, à la garder : selon eux, l'institution avait été sacralisée par le prophète et elle ne pouvait être abolie arbitrairement par le calife $^{2}$. Pour légitimer le mariage $m u t^{\prime} a$, les juristes ja 'farites s'appuyèrent sur des hadith issus du corpus chiite et sur trois versets de la sourate des Femmes, particulièrement :

« Vous est rendu licite tout ce qui n'est pas compris (dans l'énumération) précédente. (Vous pouvez donc) satisfaire votre désir, moyennant votre bien, en "préservants", non pas en débauchés. En tant que vous jouirez de ce qu'elles

1. Elle n'est pas non plus admise par les druzes, dont les normes régissant le mariage sont particulières. Cf. l'article de l'avocat Bassâm Amîn al-Halabî, « al-drûz wa zawâj al-mut'a », al-Nahâr, 20/02/2004.

2. Cf. Madelung 1985, p. 72. Voir, pour plus de détails, HefFening 1993. 
vous accordent, donnez-leur leur salaire (nuptial) : c'est obligatoire. Point de faute pour vous à convenir d'un supplément à l'obligation ${ }^{3}$. »

Le fiqh ja 'farite définit le mariage $m u t^{\prime}$ ' comme un contrat entre un homme (marié ou non) et une femme (non mariée), oral ou écrit, conclu avec ou sans témoin, qui stipule la durée du mariage et le montant du mahr. Or, dans la pratique, seules les règles et les conditions minimales sont retenues : un contrat oral entre un homme et une femme stipulant le montant du douaire et la durée du mariage. Le montant du douaire pouvait être jadis une poignée de dates, aujourd'hui il peut consister en un don ou une somme symbolique. Quant à la durée du mariage, qui doit elle aussi être précisée pour assurer sa validité, elle n'a pas de limites, ni inférieure, ni supérieure. Les époux n'ont pas l'obligation de partager le même toit, et l'homme n'a pas à subvenir aux besoins de son épouse. Il peut, en outre, contracter autant de mariages temporaires qu'il le souhaite, simultanément. Toutefois, il y a des discussions chez les oulémas contemporains pour restreindre le nombre d'épouses. La femme, elle, n'a le droit de contracter qu'un mariage à la fois, qu'il soit temporaire ou permanent. Elle doit, dans les deux cas, respecter un délai de viduité ('idda), qui est plus court dans le cas du mariage temporaire : deux mois ou 45 jours. Comme d'autres normes islamiques, celle-ci est parfois détournée par le biais d'une « ruse » (hîla) consistant à contracter un deuxième mariage temporaire de courte durée, avec le même homme, sans qu'il y ait consommation, puis d'en contracter un troisième sans devoir respecter la période de viduité, puisqu'il n'y a pas eu de consommation lors du précédent. Enfin, l'époux doit forcément être chiite, mais l'épouse, elle, peut être une kitâbiyya, c'est-à-dire chrétienne, juive ou zoroastrienne ${ }^{4}$.

Pendant la période de formation de l'islam, la pratique du mariage mut ' $a$ était liée, d'une part, au commerce caravanier qui menait, pendant de longues périodes, les hommes loin de chez eux, et, d'autre part, aux guerres. Elle est d'ailleurs aujourd'hui encore associée à des conditions économiques difficiles, ou à la guerre.

Par la suite, elle fut liée aux pèlerinages sur les lieux saints du chiisme, notamment les mausolées où sont inhumés les imâms et les descendants du prophète (ahl al-bayt), qui impliquent des mouvements et des brassages de population. Ce sont les endroits où, jusqu'à présent, il est le plus aisé de trouver

3. Coran, IV : 24. Selon la traduction de Jacques Berque, Albin Michel, Paris, 1995. D'anciennes lectures du Coran, qui n'ont pas été admises par les sunnites, ajoutent à la fin du verset : «pour un temps déterminé » (HeFFENING 1993).

4. Il y a des divergences entre oulémas à ce sujet. Voir l'entretien du cheikh al-Khichn, qui dirige le bureau des fatwas de Muhammad Husayn Fadallâh, au journal al-Balad, 25/10/2004. 
une femme à épouser pendant une période déterminée. Le fait est attesté par des voyageurs qui séjournèrent dans les villes saintes d'Irak, notamment Najaf, à partir de la fin du XIX ${ }^{\mathrm{e}}$ siècle. Là, un véritable « service » était organisé par des intermédiaires qui se chargeaient de trouver une femme pour le pèlerin célibataire ou venu sans épouse ${ }^{5}$. Il en était de même en Iran, où le mariage temporaire était courant autour des mausolées, et souvent considéré comme une forme de prostitution, par ailleurs attestée à la cour safavide d'Ispahan, par le chevalier Chardin, dès le XVII ${ }^{\mathrm{e}}$ siècle ${ }^{6}$. Néanmoins, il faut se méfier des descriptions caricaturales, simplistes ou fantasmées sur le mariage de plaisir autour des lieux sacrés, qui est parfois tout à fait assumé par les femmes, comme l'a montré l'enquête d'une anthropologue iranienne ${ }^{7}$, ou encore tout simplement un mode de vie et de subsistance, en allant d'union en union, dans l'Iran de la République islamique ${ }^{8}$.

Si l'on a souvent vu, dans ce type de mariage, une simple location du corps de la femme, c'est que l'objectif visé est, d'abord, de permettre à l'homme d'assouvir ses besoins sexuels. Dans les doctrines de l'islam, ceux-ci sont considérés comme naturels, et il faut les satisfaire pour éviter les débordements sociaux; seuls les grands mystiques sont capables d'abstinence. En outre, le besoin de l'homme de multiplier les relations sexuelles et les partenaires est reconnu. L'institution du mariage temporaire a donc pour but d'éviter l'adultère et la fornication (zinâ). À toutes les époques, les oulémas ont fait une claire distinction entre le mariage permanent, dont l'objectif est de procréer et de fonder une famille, et le mariage temporaire qui vise, lui, à satisfaire un besoin, un plaisir sexuel. Toutefois, les enfants susceptibles de naître de cette union jouissent des mêmes droits que les autres concernant l'héritage. Cette disposition marque bien la volonté d'éviter les enfants illégitimes, que la société récuse, et de dissocier le mariage temporaire de la prostitution. Toutefois, il demeure aisé, pour un homme, de renier un enfant issu d'un mariage mut 'a, d'autant que celui-ci n'est pas enregistré au tribunal religieux. Ainsi, il fut considéré comme une sorte de mariage inférieur dans la loi ja'farite.

Le mariage $m u t^{\prime} a$ pose problème ; il est déprécié, voire décrié. Vivement condamné par les sunnites, il constitua longtemps une pierre d'achoppement dans le dialogue intermusulman qui tente de rapprocher les branches sunnite et chiite de l'islam. C'est qu'il est lié à une série de points de discorde fondamentaux : l'histoire et l'interprétation du texte coranique, la critique du

5. NAKASH 1994, p. 166.

6. RichaRd 1991, p. 205-206.

7. HAERI 1989, p. $97 s q$.

8. Voir le récit de vie que fait Mohsen MaKhmaLbÂF (2003) dans un roman où il raconte le quotidien de plusieurs femmes démunies partageant une maison à Téhéran, dont une qui a vécu de sighe en sighe. 
hadîth, et l'histoire politique des débuts de l'islam ${ }^{9}$. En outre, il touche à des questions de morale et d'honneur. Déjà, en Irak, à la fin du XIX siècle, des oulémas salafistes, au vu du développement du chiisme en Irak, accusèrent les prédicateurs chiites d'avoir converti les tribus en permettant à leurs chefs de multiplier le nombre de leurs épouses, par le biais du mariage de plaisir. Le sujet allait opposer chiites et sunnites tout au long du $\mathrm{Xx}^{\mathrm{e}}$ siècle, et il est encore débattu dans les polémiques entre salafistes et chiites qui se déroulent aujourd'hui sur des sites internet. Rachîd Ridâ (m. 1935), l'animateur de la revue al-Manâr, entretint, un temps, la polémique ; des articles parurent, en réponse, dans la revue chiite al-'Irfân, publiée à Saïda, puis des traités répondirent à d'autres attaques ${ }^{10}$. Un clerc chiite du Jabal 'Âmil (l'actuel Liban-Sud), Muhsin al-Amîn (m. 1952), s'expliqua, à plusieurs reprises, pour contrer Rachîd Ridâ (AL-AMÎN 1910). Il défendit le mariage mut' $a$ en montrant la légalité de cette union au regard du fiqh ja'farite, mais relativisa sa portée en disant, en substance : ce n'est pas parce que le mariage de plaisir est permis par la Loi qu'il est couramment pratiqué chez les chiites. Bien plus, il admit que le mariage de plaisir était considéré comme honteux ('ayb) ${ }^{11}$. Il se faisait là l'écho d'un jugement moral de la société, et exprimait une gêne, de la part de certains oulémas chiites face à cette pratique. D'autres auteurs, extérieurs à la société locale, en témoignèrent.

En 1860, un voyageur, Wortabet, notait déjà : « (...) ils évitent ces mariages autant que possible, pour échapper aux sarcasmes et la réprobation des autres sectes - surtout des Mahométans [i.e. les sunnites] ${ }^{12} \ldots$ » Un rapport sur le Liban-Sud rédigé en 1937 faisait état du mariage de plaisir en précisant: « de telles unions sont assez fréquentes même parmi les chefs religieux. Cependant, les notables préfèrent tenir de tels mariages secrets et une femme de bonne famille s'y refuserait. Les mariages temporaires sont une source de conflit, notamment en ce qui concerne les enfants qui en naissent et que le père présumé refuse de reconnaître. Les tribunaux de Tyr et de Saïda ont fréquemment à connaître de tels différends ${ }^{13}$. »

Le mariage $m u t$ ' $a$ était donc dévalorisé par les chiites eux-mêmes, et ils le pratiquaient de manière discrète, pour sauvegarder tant leur image face aux sunnites que la réputation et l'honneur des familles. Toutes les contradictions dues à la tension entre théorie - c'est-à-dire droit islamique - et pratiques

\footnotetext{
9. ENDE 1985, p. 192.

10. Mervin 2000, p. 290-292.

11. MERVIN 2000, p. 313.

12. Wortabet 1860, p. 278.

13. DARCHÉ 1937, p. 21.
} 
sociales étaient déjà là. Le mariage de plaisir, survivance des temps obscurs de la jâhiliyya, était hors normes dans une société empreinte d'un rigorisme moral, qu'il fût lié aux valeurs religieuses (akhlâq) ou aux codes sociaux. Le fait qu'il fallait donner de la publicité à la doctrine pour la défendre des attaques sunnites ne faisait qu'attiser ces contradictions. Pour les oulémas chiites, le mariage permanent restait la norme idéale, à laquelle il fallait se conformer, le mariage temporaire n'étant qu'une sorte de mariage inférieur.

Les débats entre oulémas sur la modernité importée d'Europe et la nécessité d'y accommoder l'islam donnèrent l'occasion aux chiites de passer d'une attitude défensive à une position plus offensive. À partir des années 1920, ils trouvèrent matière à faire l'apologie de leur doctrine en soutenant que le mariage mut' $a$ avait précédé les nouvelles conceptions du mariage dont on débattait en Occident : mariage civil mais aussi mariage à l'essai. Un clerc chiite cita le philosophe britannique Bertrand Russell (1872-1970), auteur de l'ouvrage Le Mariage et la morale (1929) ${ }^{14}$. Aujourd' hui, les oulémas libanais et les défenseurs du mariage temporaire font toujours référence au mariage à l'essai, ou même au PACS français.

Le discours des oulémas sur le mariage temporaire était plus tourné vers les milieux cléricaux que vers la société, qui n'y était pas favorable. Tant dans les pays arabes qu'en Iran, cette forme de mariage était souvent considérée comme une forme de prostitution au sein de la société. Les points de vue formulés sur la question par les idéologues de la révolution iranienne, puis l'encouragement de la pratique par le régime islamique, marquèrent un tournant. C'est Mortaza Motahhari qui s'exprima le plus amplement sur le sujet, avec une approche nouvelle de la part d'un clerc. Le fait est d'autant plus important à souligner que Motahhari, assassiné quelques mois après la révolution, en mai 1979, fut, avec Khomeini, l'idéologue du régime islamique, et reste une référence pour les mouvements islamiques chiites - dont le Hezbollah, au Liban. L'un des plus fameux ouvrages de Motahhari, intitulé Les droits de la femme en islam, contient un chapitre sur le mariage temporaire ${ }^{15}$.

Après une brève présentation des règles, d'ailleurs simples, qui régissent ce mariage, l'auteur le place dans le contexte de la vie d'aujourd'hui. Il explique que ni les filles ni les garçons, lorsqu'ils se trouvent sous « la lourde pression de besoins instinctifs », n’ont la maturité sociale nécessaire au mariage permanent et à la fondation d'une famille. Un homme ne peut être indépendant économiquement, ayant achevé ses études, avant 25 ans, et il lui faut encore

14. ENDE 1980, p. $18 s q$.

15. MotahHari 1993. Cet ouvrage, traduit en plusieurs langue, est aussi disponible sur internet. Cf., par exemple, www.mutaa.com ou www.shia.com. 
3 ou 4 ans pour pouvoir se marier et s'installer. De même pour une femme qui étudie. Si vous demandez, écrit Motahhari, à un jeune de 17 ans, dont le besoin sexuel est à son point culminant, de se marier, les gens vont se moquer de vous. Pareil pour une étudiante de 16 ans. Or, poursuit-il, la nature n'est pas préparée pour retarder la puberté ou le besoin sexuel jusqu'à ce qu'ils aient terminé leurs études... Il leur reste donc soit l'abstinence, néfaste à la santé psychique, soit le « communisme sexuel », c'est-à-dire la multiplication des partenaires, qui mènerait l'un et l'autre en enfer, main dans la main. En effet, Motahhari considère qu'homme et femme sont égaux, et que, dans la même situation, ils adoptent le même comportement : s'il y a multiplication des partenaires, c'est de la part des deux. Pour lui, le mariage temporaire est la solution à ce problème. D'autant que comme la femme doit se limiter à un seul époux, il est évident à ses yeux, selon la même logique que précédemment, que cela implique la même limitation pour l'homme.

C'est une conception idéale du mariage mut 'a qu'il nous livre là, soustendue par une vision non moins idéale des rapports égalitaires entre les deux sexes ; celles-ci ont imprégné les milieux islamiques contemporains. Le contrat de mariage temporaire fut inclus dans le code de la République et vanté par Khomeini et par le régime. Pendant la guerre entre l'Iran et l'Irak, les veuves des martyrs étaient incitées à épouser des soldats de cette manière. En outre, l'Iran encouragea l'enregistrement des mariages temporaires au tribunal. Une sociologue effectua une enquête sur ce phénomène, à partir d'un échantillon de dix-huit cas d'enregistrement au tribunal. Sur ce nombre, dix consistaient à enregistrer un mariage temporaire en second mariage permanent (ce qu'ils n'avaient pas pu faire car ils s'étaient unis avant 1979); deux à transformer un mariage temporaire en mariage permanent. Les couples concernés étaient d'accord dans cette démarche, vivaient déjà maritalement et avaient fondé une famille. Quant aux cas restant, il s'agissait de litiges portés devant le tribunal par la femme, qui demandait l'enregistrement d'un mariage temporaire après la naissance d'un enfant ${ }^{16}$.

Suivant l'exemple de l'Iran, des clercs libanais ont appelé à enregistrer les mariages temporaires au tribunal religieux, afin de protéger les femmes et de préserver les droits des enfants qui naîtraient de ces unions, puisqu'il serait ainsi plus difficile au père de nier sa paternité ${ }^{17}$. L'un d'entre eux, qui a exercé dans un tribunal ja'farite, déclare ne pas avoir eu à enregistrer ce type de mariage, et « pense » que d'autres que lui l'ont fait, mais que les cas

16. MiR-Hosseini 1983, p. 162-163.

17. C'est le cas de Muhammad Husayn Fadlallâh. 
demeurent rares ${ }^{18}$. Seule une enquête systématique pourrait permettre d'évaluer précisément le phénomène, qui semble limité. Les efforts des clercs n'ont pas les effets escomptés sur la société, et le mariage temporaire est toujours très déconsidéré. Le débat, néanmoins, a pris une autre tournure puisque des clercs le défendent publiquement, notamment dans la presse, dans une perspective moderniste comme celle de Motahhari. Ainsi, ils s'adressent aux jeunes alors que, jusqu'à présent, le mariage temporaire était plutôt réservé aux veuves et aux divorcées ${ }^{19}$. Parallèlement, alors que l'âge du mariage permanent recule, les jeunes sont attirés par le mariage temporaire et il semble qu'ils le pratiquent de plus en plus dans les milieux étudiants, afin de concilier liberté et légalisme religieux. Dans les cercles islamiques, il est présenté comme un instrument de libération de la femme : « le chiisme a donné l'entière liberté sexuelle à la femme avec le mariage de plaisir » m'a déclaré un jeune militant. Il constitue aussi, comme en Iran, un moyen de subvertir la ségrégation sexuelle qui est de rigueur dans ces milieux.

Le fait que le discours public sur le mariage temporaire ne concerne plus seulement les femmes divorcées ou les veuves, mais s'ouvre sur les jeunes qui n'ont pas été mariées renvoie à deux problèmes : le tabou de la virginité et la question de l'autorisation du père, nécessaire, ou non, selon les avis des oulémas. Rappelons, tout d'abord, que la plupart des marja', qui sont les références religieuses des chiites, interdisent aux jeunes gens de se fréquenter avant le mariage, tout au plus leur accordent-ils de se «voir» pour faire connaissance ${ }^{20}$. Ils continuent par ailleurs à prescrire d'exclure les jeunes filles vierges des mariages temporaires, alignant leurs positions sur les normes sociales conservatrices. Certains, après Motahhari, exprimèrent des avis plus ouverts, qui se veulent à l'écoute de la jeunesse et de ses problèmes, comme pour mieux les tenir écartés du processus de sécularisation qui touche les sociétés musulmanes.

Muhammad Husayn Fadlallâh est de ceux-là. Ce clerc libanais, qui est un marja', se distingue par ses avis réformistes et modernistes, notamment en matière de bioéthique et pour ce qui concerne les questions afférant aux femmes. Il considère que celles-ci doivent occuper l'espace public et jouer un rôle social, leur reconnaît le droit de choisir leur époux (et de le demander en mariage), s'insurge contre les crimes d'honneur et désacralise

18. Entretien avec sayyid Muhammad Hasan al-Amîn, Saïda, 08/06/2006.

19. Voir, par exemple, l'entretien du cheikh Khichn dans al-Balad, art. cit.

20. Cf. AL-MAнmÛDî 2004, p. 253-254. Les fatwas prises en compte sont celles de Sistani, de Khamenei et d'autres marja' iraniens. 
la virginité. En effet, même s'il estime qu'une première expérience sexuelle avec son époux est préférable, il explique que la virginité a été hissée au rang de valeur par les traditions sociales, et non par la religion. En outre, ayant observé le problème du célibat prolongé des femmes, il préconise le mariage même dans le cas où les conditions matérielles ne sont pas satisfaites aux yeux des parents. Pour lui, la femme pubère et adulte (bâligha râchida) n'a pas besoin de l'autorisation de son père ou de son tuteur pour se marier; il en est donc théoriquement de même pour un mariage temporaire. Or, c'est l'intéressée elle-même qui sait si elle a atteint la maturité, c'est-à-dire la faculté de discerner ses intérêts de ce qui peut lui causer du tort. Attaqué pour ces positions, notamment la possibilité, pour une fille vierge, de contracter un mariage temporaire sans l'accord de son père, Fadlallâh dut s'expliquer et préciser qu'il ne l'encourageait pas, et mettait les jeunes filles en garde contre les conséquences d'un acte irréfléchi (c'est-à-dire être enceinte sans être mariée de façon permanente). Pour lui, si la société était plus ouverte, les jeunes filles n'auraient pas besoin de recourir à cette «solution » qu'est le mariage temporaire. Bien plus, selon lui, lorsque la coutume rejette ce que l'islam perçoit comme naturel, il ne faut pas suivre la coutume. Néanmoins, il estime que la vision négative d'une pratique comme le mariage temporaire dans la société peut déshonorer à la fois l'homme et la femme. Ainsi, il faut bien y réfléchir avant de profiter de la fatwa qui le permet : dans le cas d'une fille vierge, le mariage temporaire est à éviter (makrûh $)^{21}$. Un clerc proche du marja 'explique ainsi que l'autorisation du père n'est pas nécessaire, elle est préférable (afdal) ${ }^{22}$. Or, on peut se demander comment des jeunes gens iraient demander son accord pour un mariage temporaire au père d'une jeune fille ! La situation paraît tout à fait improbable. À cela, il répond que les jeunes gens peuvent demander l'autorisation pour un mariage permanent et, en attendant, contracter sans l'annoncer un mariage temporaire.

Muhammad Husayn Fadlallâh ne renie pas pour autant ce qu'il a défendu par ailleurs, et il insiste, comme d'autres que lui, sur la distinction entre l'union libre pratiquée en Occident et le mariage temporaire. Celle-ci réside dans le fait qu'il s'agit ici d'un contrat ('aqd) qui n'est pas seulement un accord entre deux personnes, mais comporte des conditions et des devoirs ; aussi prône-t-il l'enregistrement de ces mariages par les tribunaux religieux ${ }^{23}$.

21. Fadl Allâh 2001 et 1995. Voir aussi Bayynat.org, son site, qui publie Ta'ammulât et des fatwas (en arabe, français et anglais).

22. Entretien avec le cheikh Ridâ Farhat, Beyrouth, Hârat Hurayk, 27/04/2006.

23. Cf. le cheikh Khichn, al-Balad, art. cit. Entretien de M. H. Fadlallâh, Nahâr al-chabâb, 05/07/2007, repris sur son site Bayynat.org. 
En outre, il y a « sacralité » de cet accord ${ }^{24}$. Aussi les clercs continuent-ils de défendre ce mariage, légal au regard de la loi religieuse, contre les « tabous sociaux » (hurma ijtimâ 'iyya) d'une société patriarcale et masculine ${ }^{25}$. Celleci, à leurs yeux, n'est pas prête. C'est ce que confirme un fidèle appartenant à ce que l'on appelle le milieu islamique (al-hâla al-islâmiyya) : «le mariage temporaire implique une vraie relation de confiance et de compréhension mutuelle (takâmul), qui ne peut se nouer qu'au sein d'une société vraiment islamique, et la nôtre ne l'est pas. » On revient à la vision idéalisée d'une société islamique.

Autre argument en faveur du mariage temporaire: la sollicitation permanente des images à connotation sexuelle, dans l'espace public, qui perturbent notamment les jeunes, au point qu'ils ne peuvent plus contrôler leurs besoins. Enfin, l'ultime argument susceptible de toucher les jeunes est que ce mariage n'aboutit pas forcément à des relations sexuelles, puisque les deux époux s'entendent, dans le contrat qu'ils passent ensemble, sur la nature et les limites de leur liaison. Cela leur permet de se fréquenter avant d'établir un mariage permanent, ou bien d'y renoncer s'ils ne s'entendent pas. Il semble d'ailleurs que les jeunes des milieux estudiantins y soient assez sensibles, et que le mariage temporaire s'y développe. Toutefois, il est impossible d'évaluer le phénomène, puisque ce mariage reste secret. Il est, d'un côté, présenté comme marginal ou bien répandu dans d'autres communautés chiites ; ainsi, au Liban, on vous dira qu'il est pratiqué surtout en Iran. D'un autre côté, il est présenté comme en plein développement parmi les jeunes, dans des sociétés qui se modernisent, mais où les mentalités restent conservatrices. En outre, des représentations fantasmées, qui exagèrent et dénaturent le phénomène, émanent de l'extérieur des communautés chiites. C'est pourquoi les études effectuées sur le sujet doivent se contenter de relever les tensions, voire les contradictions entre la loi religieuse et les normes sociales. C'est ce que fit Linda Walbridge dans un article sur des chiites de Deaborn, dans le Michigan, où elle fait état de la tension entre religion et culture populaire ${ }^{26}$.

Deux sites internet prônent et facilitent la pratique du mariage temporaire. L'un, en persan, s'adresse dorénavant à ses seuls abonnés ${ }^{27}$; l'autre est accessible à tous et en plusieurs langues (anglais, néerlandais, ourdou, arabe et persan) ${ }^{28}$. Toutes les informations sur les modalités pratiques à suivre $\mathrm{y}$

24. Entretien avec M. H. al-Amîn (cf. note 18).

25. L'expression est de M. H. al-Amîn (cf. note 18).

26. WALBRIDGE 1997.

27. Ce site, www.sighe.com, semble organisé par des Iraniens à l'étranger.

28. Il s'agit de www.mutah.com 
sont disponibles, ainsi que des ouvrages en ligne sur le sujet, des fatwas et des témoignages. Réels ou non, ceux-ci sont très typiques. Côté masculin, ce sont des étudiants résidant en Angleterre qui se disent sexuellement frustrés, et donc perturbés dans leur travail et leur vie quotidienne, mais qui n'ont pas les moyens de fonder une famille ; ils cherchent donc à contracter un mariage temporaire pour répondre à leur détresse. Un autre explique avoir contracté un mariage temporaire pour pouvoir tenir une conversation avec une jeune fille sans avoir de doute sur la licéité de son comportement (concernant les propos ou les regards échangés). Un homme explique avoir contracté trois mariages temporaires, par le biais d'internet, afin de trouver une épouse pour se marier de façon permanente. Le quatrième est un divorcé de 21 ans qui déplore l'accroissement du taux de divorce et en déduit que l'institution du mariage permanent est en voie d'extinction. Pour lui, puisque les musulmans ont l'esprit « embrumé par la pollution de l'endoctrinement occidental », ils sont plus à même d'avoir recours au mariage temporaire qui leur garantit leur indépendance tant personnelle qu'économique, et s'engager ensuite, en connaissance de cause, dans un mariage permanent où ils appliqueraient « les valeurs islamiques pures ». Côté féminin, un seul témoignage est présenté, celui d'une enseignante du Colorado, convertie, qui raconte l'expérience assez amère du mariage temporaire qu'elle a contracté lorsqu'elle était étudiante - et encore chrétienne - avec l'homme qui devint par la suite son époux permanent. Toute la difficulté, pour elle, avait été de vivre cette union, présentée comme légale au regard de la loi religieuse, en secret.

Créé en 2000, ce site est jumelé avec un autre, qui vise à promouvoir le mariage permanent pour contrer les attaques des ennemis de l'islam dans leur entreprise de destruction de la famille par sa division en individus. Il s'agit donc d'une plate-forme permettant de trouver un époux ou une épouse ${ }^{29}$. De même, mutah.com avait organisé un service de rencontres sur internet afin d'aider hommes et femmes à trouver un partenaire pour un mariage temporaire, dans un « objectif halâl ». Toutefois, il semble que ce service ait été dérangé par des intrusions incongrues. Les organisateurs, après avoir consulté Dieu par une istikhâra (bibliomancie par le Coran), décidèrent de fermer le service, en $2004{ }^{30}$. Rien n'est dit, dans les explications fournies sur le site, sur les raisons précises qui ont semé le doute chez les animateurs du site, au point de les amener à effectuer cette consultation divine. On peut seulement supposer, entre autres, que le site a été l'objet de critiques de la part

29. Cf. www.nikahmarriage.com

30. Une dernière consultation du site juste avant la mise sous presse nous a permis de constater que la rubrique « chat » avait été rouverte (01/07/2008). 
d'internautes sunnites versés dans les polémiques sur la toile. Celles-ci sont en effet très vives, notamment entre chiites et salafistes. Le mariage temporaire reste l'une de leurs cibles.

Cependant, comme le souligne un clerc chiite libanais, la preuve de l'utilité du mariage temporaire, c'est que les sunnites, qui le critiquent, ont récemment adopté une forme de mariage comparable, le mysiar ${ }^{31}$. Selon lui, même si ce mariage est permanent, il ressemble à un mariage temporaire, vu les conditions dans lesquelles il est pratiqué. Le cheikh Yûsuf al-Qaradâwî, autorité sunnite de renom, a d'ailleurs émis une fatwa légalisant le mysiar. Il s'agit d'un contrat dans lequel la femme renonce à certains des droits qui sont ceux du mariage islamique habituel, parce qu'elle trouve des difficultés à se marier. Ainsi, elle accepte que son époux ne subvienne pas à ses besoins, ni ne vive sous le même toit. Ce type de mariage n'a rien de nouveau, indique Qaradâwî, et il est très similaire à ce que l'on appelle le mariage 'urfí, qui n'est pas enregistré au tribunal. En fait, le point commun entre ces unions, c'est qu'elles sont le plus souvent tenues secrètes, car mal acceptées par la société. Qaradâwî note à ce propos qu'il y a une différence entre « islamiquement valide » et « socialement acceptable ». Lui-même, d'ailleurs, souligne qu'il ne soutient pas le mysiar, mais le rend licite ${ }^{32}$. C'est donc la même tension que celle que nous avons relevée dans le cas du mariage temporaire, avec la différence que les oulémas chiites, pour des questions afférant à l'affirmation de l'identité chiite, défendent le mariage temporaire dans une perspective apologétique, alors que les oulémas sunnites n'ont pas besoin de le faire pour des types de mariages similaires. Ce qui explique la position d'un clerc chiite, nous déclarant: «Je crois au mariage temporaire et je le défends mais, comme d'autres, je n'accepterais pas que mes filles se marient ainsi... »

\section{Bibliographie}

Al-Amîn M., 1910 : al-Husûn al-manî'a fî al-radd 'alâ Sâhib al-Manâr [Les forteresses imprenables : réplique au directeur d'al-Manâr], Damas : matba'at al-islâh, 1328/1910.

DARCHÉ J., 1937 : Les chiites du Liban, rapport du CHEAM n 108.

ENDE W., 1980 : « Ehe auf Zeit (mut'a) in der Innerislamischen Diskussion der Gegenwart », Die Welt des Islams 20/1-2, p. 1-43.

31. J'utilise ici la transcription la plus répandue du terme.

32. Voir la fatwa de Qaradâwî sur www.islamonline.net, 29/08/2004. Sur les mariages 'urfì et mysiar, cf. EUM 2004-2005 et JURDI 2001. 
ENDE W., 1985 : « Sunniten und Schiiten in 20. Jahrhundert », Saeculum 36, p. 187-200.

Eum I., 2004-2005 : «'Urfi Marriage, an Egyptian Version of Cohabitation? », al-Raida XXIXXII, $\mathrm{n}^{\text {os }}$ 106-107, été-automne, p. 64-69.

FADL AlLÂH M. H., 1995 : al-Mar'a bayna wâqi ihâ wa haqqihâ fỉ al-ijtimâ ' al-siyâsî al-islâmî [La femme entre la réalité de sa situation et son droit dans la société politique islamique], Beyrouth : dâr al-thaqalayn.

FADL AllâH M. H., 2001 : Ta'ammulât hawla al-mar'a [Considérations autour de la femme], Beyrouth : dâr al-malâk.

HAERI S., 1989 : Law of Desire. Temporary Marriage in Iran, Londres : I.B. Tauris.

HefFenING W., 1993 : «Mut‘a », Encyclopédie de l'islam, vol. VII, 2édition, Brill, p. 758761.

JURDI S., 2001 : « Misyar Marriage », al-Raida XVIII-XIX, nº 93-94, printemps/été, p. 58-60.

Madelung W., 1985 : «Shi‘i Attitudes Toward Women as Reflected in fiqh», dans W. Madelung, Religious Schools and Sects in Medieval Islam, Londres : Variorum Reprints, chap. XII.

AL-Maнmû́î M., 2004: Ahdâth al-fatâwâ [Les dernières fatwas], Beyrouth: al-dâr alislâmiyya.

Makhmalbâf M., 2003 : Le Jardin de cristal, trad. par V. Despagnet, Paris : Le Serpent à Plumes.

Mervin S., 2000 : Un réformisme chiite. Ulémas et lettrés du Jabal 'Âmil (actuel Liban-Sud) de la fin de l'Empire ottoman à l'indépendance du Liban, Paris : Karthala-CERMOCIFEAD.

Mir-Hosseini Z., 2000 : Islam and Gender: The Religious Debate in Contemporay Iran, Londres : I.B. Tauris.

Mir-Hossein Z., 1983 : Marriage on Trial: A Study of Islamic family Law. Iran and Morocco Compared, Londres : I.B. Tauris.

Mir-Hossein Z., 1998 : «A response to Shahla Haeri's Review of Marriage on Trial », IJMES 30/3, p. 469-471.

Motahhari M., 1993 : Les droits de la femme en Islam, Paris : éd. Abbas Ahmad al-Bostani.

NAKash Y., 1994 : The Shi is of Iraq, Princeton : Princeton University Press.

Richard Y., 1991 : L'islam chi'ite. Croyances et idéologies, Paris : Fayard.

Walbridge L., 1997 : «Sex and the Single Shi'ite: Mut'a Marriage in an American Lebanese Shi'ite Community », WLUML, dossier 18, octobre.

Wortabet J. M. D., 1860 : Researches into the Religions of Syria, Londres : James Nisbet.

Zouari F., 2001 : « Mariage de saison », Jeune Afrique l'intelligent 2097, 20-26 mars, p. 39. 